\title{
LAS NUEVAS TECNOLOGÍAS EN LA LEGISLACIÓN TRIBUTARIA MEXICANA. ESPECIAL REFERENCIA AL MODELO DE GESTIÓN TRIBUTARIO
}

\author{
Enrique Pons Franco*
}

\section{RESUMEN}

El objeto del presente documento, como su nombre lo indica, tiene como finalidad realizar una crítica, a guisa de ensayo, breve, y seguramente insuficiente por los amplios contenidos que incluye, sobre la incorporación de las nuevas tecnologías en la legislación gestión tributaria mexicana, especialmente referida al Código Fiscal de la Federación, la cual, como daremos cuenta, incluye la remisión de información contable de los contribuyentes por medios electrónicos. La principal hipótesis de nuestro trabajo se basa en descubrir: ¿De qué manera se integraron al ordenamiento jurídico tributario las nuevas tecnología, particularmente, la relativa a los medios electrónicos; ¿Realizó realmente el legislador un trabajo parlamentario agudo, o simplemente, la incorporación de los medios electrónicos pasó sin mayor debate en su proceso de aprobación?; y por último, verificar si la incorporación de medios electrónicos en el sistema jurídico que regula la gestión del tributo en México reúne los requisitos de carácter constitucional. Todas éstas hipótesis, claro está, en lo que hace al fenómeno de la gestión tributaria, concepto el cual, también nos ocupamos de definir al menos de manera orientadora. Asimismo, como suele acontecer en todas las investigaciones de tipo jurídico, cabe señalar que esta será de tipo documental y dogmático, teniendo especial referencia, en los criterios emitidos por la Suprema Corte de Justicia de la Nación.

Palabras claves: Tributo. Gestión. Medios. Electrónicos.

*Doctor en Derecho. Profesor Investigador. División Académica de Ciencias Económico Administrativas. Universidad Juárez Autónoma de Tabasco.

DIRECCIÓN PARA RECIBIR CORRESPONDENCIA: Enrique Pons Franco correo electrónico: epons@romeroponsabogados.mx 


\section{ABSTRACT}

The purpose of this document, as its name suggests, aims to critique, by way of trial brief, and certainly insufficient for extensive information includes, on the incorporation of new technologies in managing Mexican tax law, especially referring to the Tax Code of the Federation, which, as we realize, includes the remission of accounting information from taxpayers electronically. The main hypothesis of our work is to find out: How did the tax law integrate new technology, particularly relative to electronic media?; Do we really conducted the legislature an acute parliamentary work, or simply incorporating electronic media passed without further debate in its approval process?; and finally, determine whether the incorporation of electronic media in the legal system governing the management of tribute in Mexico meets constitutional requirements. All these hypotheses, of course, what makes the phenomenon of tax management concept which also take care to define at least guiding way. Also, as often happens in all investigations legal, it should be noted that this document be dogmatic type, with special reference to the criteria issued by the mexican Supreme Court of Justice of the Nation

Key words: Tax. Managing. Electronic. Media. 
Aún hoy, con la constante evolución del derecho tributario, el Código Fiscal de la Federación ${ }^{1}$ (CFF) sigue siendo un elemento indispensable para lograr un orden en la gestión tributaria, es la herramienta fundamental y piedra angular que regula toda una serie de aspectos relativos a las relaciones entre la administración tributaria y los contribuyentes, la cual, en México es realizada por la Secretaría de Hacienda y Crédito Público $^{2}$ (SHCP), por conducto del Servicio de Administración Tributaria ${ }^{3}$ (SAT).

Ahora bien, concretamente en el campo relacionado con la utilización de las nuevas tecnologías informáticas en la gestión tributaria, nos encontramos ante la urgente necesidad que los avances en todas las áreas relacionadas con el tema que aquí nos ocupa, se adecuen a los principios constitucionales que regulan la relación jurídicotributaria, tales como legalidad, seguridad jurídica, proporcionalidad, equidad, capacidad contributiva, todos, visibles en el artículo 31, fracción IV de la Constitución Política de los Estados Unidos Mexicanos (CPEUM), puesto que de otra forma, como bien ha apuntado (Checa, 2010), se corre el riesgo que existan más efectos negativos, que positivos, al ocurrir un fenómeno de degeneración en la aplicación de los principios constitucionales antes citados.

Al hilo de lo expuesto, para los efectos que nos ocupan en el presente ensayo, nos ceñiremos a establecer que el concepto de "gestión tributaria" se refiere a las acciones que lleva a acabo la administración estadal para poder realizar un fin; es una actividad fundamental en la función administrativa, clave para la correcta recaudación de los ingresos públicos, en la que intervienen recursos técnicos y humanos, desarrollando de esta forma la interrelación con los contribuyentes. Según (Pavés, 1991), añade que la gestión tributaria tiene como eje central la participación mixta del obligado tributario con la administración, desde que nace la obligación, hasta su total conclusión.

\footnotetext{
${ }^{1}$ Recordemos que nuestro vigente Código Fiscal de la Federación fue publicado en el Diario Oficial de la Federación el 31 de diciembre de 1981.

2 De acuerdo al artículo 4, segundo párrafo, del Código Fiscal de la Federación, la recaudación proveniente de todos los ingresos de la Federación, aun cuando se destinen a un fin específico, se hará por la Secretaría de Hacienda y Crédito Público o por las oficinas que dicha Secretaría autorice.

${ }^{3}$ Conforme a lo dispuesto en los artículos 1 y 2 de la Ley Orgánica del Servicio de Administración Tributaria, éste es un órgano desconcentrado de la Secretaría de Hacienda y Crédito Público, con el carácter de autoridad fiscal, y con las atribuciones y facultades ejecutivas que señala esta Ley, el cual tiene las responsabilidades: 1) De aplicar la legislación fiscal y aduanera con el fin de que las personas físicas y morales contribuyan proporcional y equitativamente al gasto público; 2) De fiscalizar a los contribuyentes para que cumplan con las disposiciones tributarias y aduaneras; 3) De facilitar e incentivar el cumplimiento voluntario de dichas disposiciones; y 4) De generar y proporcionar la información necesaria para el diseño y la evaluación de la política tributaria.
} 
Así las cosas, por un lado podemos ponderar que la gestión tributaria reviste el carácter de "interés público", puesto que la sociedad está interesada en que los ingresos públicos que son destinados respectivamente al gasto público, se obtengan con la máxima prontitud y eficiencia, y por tanto, no debiera existir oposición para que se incorporen nuevas tecnologías para alcanzar dichos fines públicos.

Sin embargo, de otro, debe observarse que la tecnología, no puede suplir la aplicación de los principios constitucionales que hemos señalado, los cuales, regulan la relación jurídico-tributaria. Dicho de otra forma, es la tecnología la que debe servir al derecho, y no el derecho el que debe servir a la tecnología, pues de otra forma, y como daremos cuenta páginas más adelante, se corre el grave riesgo de trastocar diversas garantías del ciudadano (contribuyente en este caso).

De lo expresado con antelación, tenemos que precisamente la materia objeto de estudio en el presente ensayo tiene como finalidad primordial poner de relieve -como su nombre lo indica- la manera en que las nuevas tecnologías se han incorporado en la gestión del tributo, dando cuenta, en primer lugar, de la manera en que los medios electrónicos se incorporaron gradualmente al CFF; para en un segundo plano, dar vista al fenómeno del envío de información de carácter contable por los citados medios electrónicos, y en este particular caso, apuntar la posible inconstitucionalidad de las disposiciones que regulan dichos actos jurídicos, para finalmente presentar un apartado de nuestras respectivas conclusiones.

Ciertamente, la gestión del tributo sobre los contribuyentes nunca ha sido una tarea fácil, particularmente, con los contribuyentes mexicanos ${ }^{5}$. No obstante, estamos seguros que en la medida que las leyes tributarias se encuentren más cercanas al cumplimiento de las garantías constitucionales, y en consecuencia, más lejanas de la arbitrariedad, en este caso, sobre la invasión de la vida privada de las personas, se podrá incidir de una mejor manera en la recaudación del tributo.

\footnotetext{
${ }^{4}$ Por ende, debemos tomar en consideración que el concepto de “interés público" justifica ciertas acciones del Estado, claro está, con ciertas limitaciones que sirven de motivación a cada acto de molestia que sobrevenga sobre la esfera jurídica de los particulares, dependiendo del área del derecho en la que nos encontremos. Vid. OCHOA HUERA, C.: "El carácter administrativo del derecho a la información” en Boletín Mexicano de Derecho Comparado, Enero-Abril, México, 2015, p. 164.

${ }^{5}$ Por demás interesante resulta el estudio presentado por DUGAIN, V. y OLABERRIA, E.: "What Makes Mexicans Happy?”, OECD Economics Department Working Papers, No. 1196, OECD Publishing, Paris, 2015, visible en: http://dx.doi.org/10.1787/5js4h5qp6l0w-en y consultado el 20 de abril de 2015, en donde se da cuenta, con base en estadísticas del INEGI, sobre las principales fuentes de felicidad del promedio de la ciudadanía en México, dentro de las cuales, claro está, no se encuentra el cumplimiento de las obligaciones tributarias.
} 


\section{LA INCORPORACIÓN DE MEDIOS ELECTRÓNICOS EN EL CÓDIGO FISCAL DE LA FEDERACIÓN}

Según la Organización para la Cooperación y el Desarrollo Económico (OCDE), México es uno de los países del entorno iberoamericano que promedia las mejores perspectivas en cuanto al rubro de desarrollo de ciencia y tecnología, incluso, tomando como referencias datos relativos al gobierno electrónico ${ }^{6}$ y los importantes avances que se han realizado en los últimos años ${ }^{7}$. Lo anterior, no es producto de la casualidad, y para ello, bien podemos remontarnos a la incorporación en el Código Fiscal de la Federación de los medios electrónicos en el año de $2004^{8}$, al establecer el Capítulo Segundo, denominado "De los Medios Electrónicos" que comprende actualmente los artículos 17-C, 17-D, 17-E, 17-F, 17-G, 17-H, 17-I y 17-J Y 17-K.

Es de relevancia señalar que en los más de 10 años que lleva vigente dicha normativa tributaria, solamente el artículo 17-D ha sido reformado o modificado en dos ocasiones, lo que denota en primer término, que las disposiciones legales que regulan los medios electrónicos en materia tributaria han permanecido con relativa estabilidad y eficiencia, aunque especial mención merece el reclamo de inconstitucionalidad del artículo 17-K, el cual, como hemos dejado constancia páginas atrás, regula el denominado "buzón tributario", tanto así, que en el mes de febrero de 2015, la Suprema Corte de Justicia de la Nación determinó, por contradicción de tesis, que resulta procedente el otorgamiento de la suspensión provisional ${ }^{9}$ contra la aplicación

\footnotetext{
${ }^{6}$ Por “gobierno electrónico”, nos señala TÉLLEZ VALDÉS, “debe entenderse el conjunto de actividades de la función pública que aprovecha las TIC para conseguir un mejoramiento en la provisión de servicios a los ciudadanos y habitantes, mediante la automatización de procesos internos de organización y funcionamiento propio de cada entidad pública, trámites en línea y la optimización de recursos tecnológicos, financieros y humanos”, Vid. TÉLLEZ VALDÉZ, J.A.: Lex cloud computing. Estudio jurídico del cómputo en la nube de méxico, Institito de Investigaciones Jurídicas-Universidad Nacional Autónoma de México, México, 2013, p. 17, disponible en http://biblio.juridicas.unam.mx/libros/7/3249/4.pdf. Consultado el 24 de abril de 2015.

${ }^{7}$ Vid. Organización para la Cooperación y el Desarrollo Económico.: Perspectivas de la OCDE sobre ciencia, tecnología e industria 2014 (Version abreviada): Informe Iberoamericano. México en 10.1787/9789264226487-10-es, consultado el 19 de abril de 2015.

${ }^{8}$ Mediante Decreto publicado en el Diario Oficial de la Federación el día 5 de enero de 2004 .

9 Vid. "SUSPENSIÓN EN EL JUICIO DE AMPARO. PROCEDE CONCEDERLA CONTRA LA APLICACIÓN DE LOS ARTÍCULOS $17 \mathrm{~K}$ Y 28, FRACCIÓN IV, DEL CÓDIGO FISCAL DE LA FEDERACIÓN (LEGISLACIÓN VIGENTE A PARTIR DEL 1 DE ENERO DE 2014). El análisis del proceso legislativo que culminó con la adición y modificación de los anteriores preceptos por Decreto publicado en el Diario Oficial de la Federación el 9 de diciembre de 2013 y atendiendo a lo dispuesto por el artículo 128 de la Ley de Amparo, permite establecer que procede conceder la suspensión en contra de su aplicación, al no contravenirse disposiciones de orden público ni afectarse el interés social, ya que las obligaciones que se imponen a los contribuyentes, consistentes en emplear el buzón tributario como medio de comunicación electrónica con la autoridad hacendaria y enviar de manera mensual su información contable mediante la página de Internet del Servicio de Administración Tributaria, si bien forman parte de las medidas implementadas a fin de agilizar los procesos de recaudación y fiscalización, lo cierto es que la concesión de la medida cautelar no impide que el contribuyente cumpla con sus obligaciones fiscales de presentar promociones, solicitudes y avisos e incluso desahogar los
} 
de los artículos 17-K, así como del artículo 28, fracción IV (del cual nos ocuparemos páginas adelante), ambos, del Código Fiscal de la Federación.

Lo anterior, puede anticipar, conforme a las reglas de la doctrina jurisprudencial de la "apariencia del buen derecho10", un resultado que declare inconstitucionales dichos preceptos, sin embargo, habrá que seguir de cerca el debate que se genere en nuestro Alto Tribunal cuando se resuelva en definitiva el fondo del asunto.

No obstante lo anterior, mediante la incorporación de los denominados "medios electrónicos" en el CFF, se generó una colisión tripartita entre diversas ramas del derecho y la tecnología.

De un lado, y por la materia especialísima que regula el CFF, tenemos al Derecho Tributario. De otro, por el contenido que buscan regular los "medios electrónicos" aparece el Derecho Administrativo; y en un tercer momento, la tecnología.

En ese orden de ideas, como menciona (Ponce, 2014) ${ }^{11}$, no se puede negar la relevancia que la ciencia del Derecho administrativo tiene con la tecnología y la

requerimientos que la autoridad hacendaria le formule en forma directa, ya sea mediante documento impreso o a través de otros medios electrónicos en documento digital, ni tampoco que la autoridad hacendaria ejerza sus facultades de comprobación; habida cuenta que ésta puede notificar a los contribuyentes los actos y resoluciones que emita a través de los mecanismos ordinarios previstos para tal efecto, solicitando la información contable para verificar el cumplimiento de las obligaciones a través de los procedimientos de fiscalización, como son la revisión de gabinete y la visita domiciliaria”. Época: Décima Época , Registro: 2008430, Instancia: Segunda Sala Tipo de Tesis: Jurisprudencia, Fuente: Gaceta del Semanario Judicial de la Federación Libro 15, Febrero de 2015, Tomo II, Materia(s): Común, Tesis: 2a./J. 2/2015 (10a.), Página: 1760.

${ }^{10}$ Vid. “APARIENCIA DEL BUEN DERECHO. CUESTIONES JURÍDICAS. La apariencia del buen derecho se traduce en un estudio previo de la cuestión planteada para realizar un juicio de probabilidad sobre la procedencia de lo solicitado por quien promovió el juicio de amparo. Ese análisis no implica una declaratoria de inconstitucionalidad del acto reclamado, porque ello debe ser materia de la sentencia. Ahora, cuando el peticionario de garantías hace valer un punto de derecho, sin referirse a cuestiones de hecho, su pretensión no puede ser descartada en forma superficial, aun cuando existan tesis aisladas que desvirtúen la postura de la parte quejosa. Por consiguiente, la apariencia del buen derecho no puede servir como justificante para negar la suspensión del acto reclamado, cuando el peticionario de amparo hizo valer cuestiones jurídicas que pueden ser debatidas y que, en todo caso, deben ser materia de un análisis exhaustivo en la sentencia que resuelva el caso concreto. TERCER TRIBUNAL COLEGIADO EN MATERIA CIVIL DEL PRIMER CIRCUITO. Época: Décima Época, Registro: 2001572, Instancia: Tribunales Colegiados de Circuito, Tipo de Tesis: Aislada, Fuente: Semanario Judicial de la Federación y su Gaceta , Libro XII, Septiembre de 2012, Tomo 3, Materia(s): Común, Tesis: I.3o.C.15 K (10a.), Página: $1510 ”$.

${ }^{11}$ Vid. PONCE SOLÉ, J.: “Ciencias sociales, Derecho Administrativo y buena gestión pública. De la lucha contra las inmunidades del poder a la batalla por un buen gobierno y una buena administración mediante un diálogo fructífero”, en Gestión y Análisis de políticas Públicas, Revista del Instituto Nacional de Administración Pública del Ministerio de Hacienda y Administraciones Pública de España, 
aplicación del Derecho en general, que puede, y debe, recuperar su papel en el concierto de las ciencias sociales con aportaciones sobre el diseño de políticas públicas en garantía de los derechos, del buen gobierno y la buena administración, esto es, del buen funcionamiento de lo público.

Sin embargo, con el ánimo de enriquecer la presente investigación, nos dimos a la tarea de ubicar el contenido del Diario de Debates realizado en la Cámara de Diputados el 28 de diciembre de 2003 (fecha en que se aprobó el Dictamen de la Comisión de Hacienda y Crédito Publico, con Proyecto de Decreto por el que se reforman, adicionan y derogan diversas disposiciones del Código Fiscal de la Federación ${ }^{12 ")}$, para conocer las posturas, manifestaciones y justificaciones de los legisladores federales al momento de realizar la votación nominal en la que se aprobó la incorporación de una legislación novedosa en cuanto a la gestión tributaria corresponde.

Al hilo que nos ocupa, podemos señalar que nuestra indagatoria arrojó que no existió tal debate en la Cámara de Diputados. Solamente existió un posicionamiento del diputado Manuel Pérez Cárdenas, del grupo parlamentario del Partido Acción Nacional, que reservó el artículo 17-D, segundo párrafo, del CFF, para presentar una propuesta de modificación a dicho artículo, la cual, fue votada por el Pleno de la Cámara, y siendo ésta desechada por la mayoría.

Cabe señalar, que a la postre, el artículo 17-D fue modificado, para medularmente depositar en el SAT las facultades de regulación administrativa de la firma electrónica avanzada, siendo que el artículo original las atribuyó al Banco de México. Sin embargo, no existió discusión de mayor relieve cuando se incorporó inicialmente al CFF la normatividad en materia de medios electrónicos. Lo anterior, nos indica, como señala (Fernández, 2012) ${ }^{13}$, "que las políticas de innovación tecnológica ocupan un lugar central entre las actuaciones del sector público como herramienta para impulsar

Nueva Época. Número 11, España, Enero-Junio, 2014, visible en http://revistasonline.inap.es/index.php?journal=GAPP\&page=issue\&op=view\&path\%5B\%5D=685. Consultada el 21 de abril de 2015.

12 http://gaceta.diputados.gob.mx/, consultado el 20 de abril de 2015.

${ }^{13}$ Vid. FERNÁNDEZ GONZÁLEZ, A.: "Entes instrumentales en políticas de innovación tecnológica de las administraciones públicas: La experiencia de los centros de supercomputación en España y su comparación con otros países” Gestión y Análisis de políticas Públicas, Revista del Instituto Nacional de Administración Pública del Ministerio de Hacienda y Administraciones Pública de España, Nueva Época, número $\quad 8$, julio-diciembre, 2012, $\quad$ pp. $65-97, \quad$ visible en http://revistasonline.inap.es/index.php?journal=GAPP\&page=article\&op=view\&path\%5B\%5D=10004\& path\%5B\%5=10339, consultada el 21 de abril de 2015. 
el crecimiento a largo plazo. Se componen del conjunto de actuaciones - de las administraciones públicas- tendentes a favorecer la asignación de recursos para la generación de tecnología por parte de las organizaciones con acciones dirigidas tanto a incentivar directamente la innovación empresarial como a mejorar el entorno institucional en el que se desarrollan estas actividades innovadoras".

\section{EL ENVIÓ DE INFORMACIÓN CONTABLE POR MEDIOS ELECTRÓNICOS A LAS AUTORIDADES FISCALES}

\section{a) ANÁLISIS CONCRETO DEL ARTÍCULO 28, FRACCIÓN IV DEL CÓDIGO FISCAL DE LA FEDERACIÓN Y SUS EFECTOS SOBRE LA GARANTÍA DE SEGURIDAD JURÍDICA.}

Como consecuencia de las reformas y adiciones en cuanto a "medios electrónicos" se refiere y de las cuales hemos dado cuenta páginas atrás, el legislador mexicano ponderó recientemente, en el artículo 28, fracción IV del CFF, la obligación para todos los contribuyentes, de ingresar de forma mensual su información contable a través de la página de internet del SAT y por ende, faculta a dicha autoridad fiscal a obtener la misma, sin que esta facultad se encuentre reglamentada ni encaminada a verificar el cumplimiento de sus obligaciones para contribuir al gasto público.

En primer lugar se debe precisar que la facultad de la autoridad de obtener de manera mensual la información contable de los contribuyentes a través de su página de internet es un acto de molestia para éstos, conforme al cual la autoridad fiscal tendrá acceso a sus papeles, sin embargo, consideramos que dicha facultad no se encuentre encaminada a verificar el cumplimiento de las obligaciones tributarias para contribuir al gasto público (la gestión del tributo), ni tampoco se verifica la existencia del "interés público", puesto que en nada abona el conocer de forma mensual toda la información contable de un contribuyente.

En este sentido, si las facultades de las autoridades fiscales de emitir actos de molestia a los contribuyentes, sólo se justifican si éstos se encuentran encaminados a verificar el cumplimiento de sus obligaciones para contribuir al gasto público, es claro que la facultad de la autoridad fiscal de obtener mensualmente la información contable de los contribuyentes a través de su página de Internet, de ninguna manera se encuentra justificada, ya que ello no está encaminado a verificar el cumplimiento de sus obligaciones para contribuir al gasto público. En tal virtud, es preciso mencionar 
que el propio legislador reconoce que la finalidad de la facultad de la autoridad de obtener mensualmente la información contable de las personas obligadas a llevar contabilidad, es simplificar y facilitar el cumplimiento de su obligación tributaria y de ninguna manera, fiscalizar a los contribuyentes.

En efecto, de conformidad con el Dictamen del Decreto por el que se reforman, adicionan y derogan diversas disposiciones del CFF, publicado el 9 de diciembre de 2013 en el Diario Oficial de la Federación, vigente en lo que nos interesa desde el $1^{\circ}$ de julio de 2014 , se desprende que la finalidad de las reformas propuestas relativas a llevar la contabilidad a través de medios electrónicos es simplificar y facilitar el cumplimiento de la obligación tributaria, y no así, verificar el cumplimiento de las obligaciones para contribuir al gasto público de los gobernados.

En este orden de ideas, es claro que la facultad de la autoridad fiscal de obtener mensualmente la información contable de los contribuyentes a través de su página de internet no se encuentra justificada y por lo tanto, insistimos en el presente ensayo, vulnera la garantía de seguridad jurídica establecida en el artículo 16 constitucional. Recordemos brevemente, siguiendo para tales fines a CARBONELL ${ }^{14}$, que la seguridad jurídica guarda una estrecha relación con el "estado de derecho", y aunque si bien, la idea a la cual aquí nos referimos es de carácter abstracto, esta implica la sujeción de los poderes públicos al derecho; a la posibilidad de controlar a los poderes públicos por medio del ordenamiento jurídico para estar en posibilidad de preservar la libertad, la igualdad, y los bienes de los miembros de la sociedad.

Además, de conformidad con la disposición legal en comento, la autoridad fiscal se encontrará facultada a obtener información contable de los contribuyentes, sin que sus actos cumplan con el mínimo de requisitos que necesariamente deben observar, entre otros, los establecidos en el artículo 16 de la CPEUM, esto es, que se efectúen a través de mandamiento por escrito emitido por autoridad competente, debidamente fundado y motivado.

En este sentido, si bien el legislador en casos excepcionales faculta a la autoridad fiscal para efectuar actos de molestia a los contribuyentes, ello sólo es con la finalidad de verificar el cumplimiento de sus obligaciones de contribuir al gasto público, además de que dichos actos forzosamente deben observar ciertos requisitos, para que los

${ }^{14}$ Vid. CARBONELL SÁNCHEZ, M.: Teoría de los derechos humanos, 2da. edición, Universidad Nacional Autónoma de México-Instituto de Investigaciones Jurídica, México, 2013, pp. 65-67. 
contribuyentes cuenten con certeza jurídica respecto de la forma en que deben llevarse a cabo. En el caso concreto, la facultad de las autoridades fiscales de obtener información contable de los contribuyentes, debe efectuarse de forma que los gobernados conozcan el objeto de dichos actos, su duración, la debida identificación de la autoridad que lo está llevando a cabo, así como resultado de los mismos por medio de un oficio que determine su situación fiscal, de forma que en todo momento cuenten con seguridad jurídica respecto del acto de molestia que están soportando.

Contrario a lo anterior, el artículo 28, fracción IV del CFF, facultan a la autoridad fiscal a obtener información contable de los contribuyentes, sin que dicho acto de molestia se encuentre fundado, motivado, ni regulado, por lo que su práctica quedará a su completo arbitrio.

Peor aún, dicho acto de molestia ni siquiera se encuentra acotado a un tiempo prudente para lograr el objetivo que con éste se pretende, sino que se establece que la información contable deberá ingresarse mensualmente a la página de internet del SAT, lo que ocasiona una molestia constante y una permanente intromisión en los papeles de los contribuyentes, lo que es contrario a la garantía de seguridad jurídica establecida en el artículo 16, primer párrafo constitucional, que incluso, ha generado que otras disposiciones de naturaleza análoga hayan sido declaradas previamente como contrarias al texto constitucional ${ }^{15}$.

${ }^{15}$ Vid. VISITAS DOMICILIARIAS O REVISIÓN DE LA CONTABILIDAD. EL ARTÍCULO 46-A DEL CÓDIGO FISCAL DE LA FEDERACIÓN (VIGENTE EN LOS AÑOS DE 1995 A 1997), ES VIOLATORIO DE LA GARANTÍA DE SEGURIDAD JURÍDICA CONTENIDA EN EL ARTÍCULO 16 CONSTITUCIONAL, SÓLO EN CUANTO A QUE NO SEÑALA UN LÍMITE A LA DURACIÓN DE TALES ACTOS DE FISCALIZACIÓN QUE SE PRACTICAN A DETERMINADOS GRUPOS DE CONTRIBUYENTES. Si bien es cierto que el artículo 16 de la Constitución Política de los Estados Unidos Mexicanos, en sus párrafos primero, octavo y décimo primero, establece como un derecho subjetivo público de los gobernados el que no puedan ser molestados en su persona, papeles o domicilio y la inviolabilidad de éste; también lo es que el propio precepto permite a las autoridades practicar tales actos de molestia, a fin de que cumplan con el propósito que dio origen a dichos actos, cubriendo desde luego los requisitos que debe contener todo acto de molestia o la orden de visita. Por consiguiente, debe entenderse que esos actos están delimitados temporalmente, esto es, que deben estar acotados por un tiempo prudente para lograr el objetivo que con ellos se pretende, pues de no ser así se volverían en una molestia constante o en una permanente intromisión al domicilio, lo que es contrario a la protección que otorga el citado precepto constitucional. En congruencia con lo anterior, es de estimarse que el artículo 46-A del Código Fiscal de la Federación, vigente en los años de mil novecientos noventa y cinco a mil novecientos noventa y siete, sólo en lo que establece la segunda parte de su primer párrafo, es violatorio de la garantía constitucional de referencia, en razón de que prevé una regla general de duración máxima de las visitas domiciliarias o de la revisión de la contabilidad, a la que deben ajustarse las autoridades fiscales, pero excluye de la aplicación de la misma a ciertos grupos de contribuyentes, respecto de los cuales no señala un plazo máximo de duración para los actos de fiscalización que se les practiquen, de modo que queda al arbitrio de las mencionadas autoridades la duración del acto de molestia, pudiendo, incluso, volverse indefinido o extender la conclusión de la visita, lo que es contrario a la citada garantía de seguridad jurídica. Tesis 1a. LVII/2001, Novena Época, emitida por la Primera Sala de la Suprema 
En tal virtud, es evidente que se genera al ciudadano/contribuyente un total estado de incertidumbre e inseguridad jurídica, toda vez que desconoce el uso o la finalidad que el SAT le dará a la información que le envíe a través de su página de internet, la forma y la frecuencia en que la revisará, así como si dicha información se encontrará debidamente protegida, siendo que la misma es de carácter privada y delicada.

Al respecto, es preciso señalar que actualmente las autoridades no cuentan con sistemas confiables mediante los cuales puedan custodiar adecuadamente la información que los particulares les proporcionan, por el contrario, en la práctica es de conocimiento público el mal uso que se ha dado a la misma, basta mencionar a manera de ejemplo el caso del Registro Nacional de Usuarios de la Telefonía Móvil (RENAUT), a través del cual se obligó a los usuarios de telefonía móvil de registrar sus datos, sin que actualmente dicho Registro haya sido efectivo y sin que se conozca el verdadero destino y uso de dicha información, que incluso, acarreó que el Poder Judicial de la Federación (PJF) negara el otorgamiento de la medida suspensional prevista en la Ley de Amparo, puesto que los concesionarios de la telefonía celular en México, se opusieron vía juicio de amparo, a determinados lineamientos que las autoridades de la Comisión Nacional de Protección y Defensa al Usuario de Servicios Financieros (CONDUSEF) emitieron para garantizar que los datos de aquellas personas físicas que no desearan que su información fuera utilizada para fines comerciales ${ }^{16}$.

Corte de Justicia de la Nación, visible en el Semanario Judicial de la Federación y su Gaceta, Tomo XIII, junio de 2001, página 248.

16 Vid. SUSPENSIÓN DEFINITIVA EN EL AMPARO. ES IMPROCEDENTE CONTRA LA APLICACIÓN DE LOS LINEAMIENTOS POR LOS QUE SE DETERMINA LA OPERACIÓN Y FUNCIONAMIENTO DEL REGISTRO PÚBLICO DE USUARIOS -PERSONAS FÍSICAS- QUE NO DESEEN QUE SU INFORMACIÓN SEA UTILIZADA PARA FINES MERCADOTÉCNICOS O PUBLICITARIOS, EMITIDOS POR LA COMISIÓN NACIONAL DE PROTECCIÓN Y DEFENSA AL USUARIO DE SERVICIOS FINANCIEROS, PORQUE DE CONCEDERSE SE SEGUIRÍA PERJUICIO AL INTERÉS SOCIAL Y SE CONTRAVENDRÍAN DISPOSICIONES DE ORDEN PÚBLICO. El artículo 124, fracción II, de la Ley de Amparo condiciona la concesión de la suspensión al hecho de que no se siga perjuicio al interés social, ni se contravengan disposiciones de orden público. Por su parte, de la interpretación de los artículos 1o. y 8o. de la Ley de Protección y Defensa al Usuario de Servicios Financieros, se colige que la finalidad primordial de dicho ordenamiento es proteger al sector de la población que utiliza los servicios financieros, estableciéndose expresamente, entre otras medidas, un registro de usuarios que no desean que su información sea utilizada para fines mercadotécnicos o publicitarios. Por tanto, atendiendo a esa finalidad primordial, es improcedente la suspensión definitiva en el amparo contra la aplicación de los mencionados lineamientos, porque de concederse se actualizarían los supuestos señalados en el citado artículo 124 que impiden su otorgamiento, ya que es preocupación de la sociedad que no se moleste con promociones y ofertas vía telefónica a quienes utilicen los servicios financieros y que los prestadores de éstos cumplan las leyes. Además, la intimidad y el respeto de la vida privada son bienes que tutela la Constitución Política de los Estados Unidos Mexicanos en sus artículos 6o. y 16, de tal forma que los indicados lineamientos se ajustan precisamente a esa directriz y efecto, lo que obviamente merece tutela judicial en tanto se tramita el juicio de garantías, dada la presunción de validez que tienen los actos de autoridad, a cuyo imperio están sujetas las entidades financieras. Época: Novena Época, Registro: 166782, Instancia: Tribunales Colegiados de Circuito , Fuente: Semanario 
De todo lo anteriormente expuesto, resulta evidente la violación a la garantía de seguridad jurídica consagrada en el artículo 16 de la CPEUM, toda vez que el artículo 28, fracción IV del CFF permite que el contribuyente sea molestado por las autoridades fiscales, sin que el acto de molestia guarde relación alguna con su obligación de contribuir a los gastos públicos, ni se encuentre fundado, motivado o reglamentado, además, que tampoco guarda ninguna relación con alguna actividad relacionada con la gestión del tributo por parte del SAT.

\section{b) EL ARTí́CULO 28, FRACCIÓN IV DEL CÓDIGO FISCAL DE LA FEDERACIÓN Y LA VULNERACIÓN A LOS PRINCIPIOS DE RAZONABILIDAD, PROPORCIONALIDAD JURÍDICA Y LEGALIDAD}

Como se mencionó anteriormente, de conformidad con el principio de legalidad establecido en el artículo 16 de la CPEUM, el legislador no puede actuar en exceso de poder, ni arbitrariamente en perjuicio de los gobernados. De dicho principio, y a los fines que aquí nos ocupan, podemos señalar, se desprenden a su vez, los de razonabilidad y proporcionalidad jurídica, que a decir de nuestra SCJN, implican que al fijar el alcance de una garantía individual por parte del legislador se debe: a) perseguir una finalidad constitucionalmente legítima; b) ser adecuada, idónea, apta y susceptible de alcanzar el fin perseguido; c) ser necesaria, es decir, suficiente para lograr dicha finalidad, de tal forma que no implique una carga desmedida, excesiva o injustificada para el gobernado; y, d) estar justificada en razones constitucionales, de cual se deduce también que el legislador debe razonada y constitucionalmente justificar el proceso legislativo de creación de la norma.

Por lo anterior, insistimos al señalar, que de conformidad con el principio de legalidad, establecido en el artículo 16 de la Constitución Política de los Estados Unidos, los actos de autoridad deben estar debidamente fundados y motivados, debiéndose entender como tal, la circunstancia de que el Congreso que expide la ley, constitucionalmente esté facultado para ello, ya que estos requisitos, en tratándose de actos legislativos, se satisfacen cuando actúa dentro de los límites de las atribuciones que la Constitución correspondiente le confiere (fundamentación), y cuando las leyes que emite se refieren a relaciones sociales que reclaman ser jurídicamente reguladas (motivación).

Judicial de la Federación y su Gaceta, Tomo XXX, Julio de 2009 , Materia(s): Administrativa, Tesis: I.4o.A.675 A, Página: 2081. 
Por otra parte, es preciso reiterar que de conformidad con dicho artículo, las personas que estén obligadas a llevar la contabilidad deberán ingresarla de forma mensual a través de la página de internet del SAT, de acuerdo con las reglas de carácter general que se emitan para tal efecto. En este sentido, del Decreto por el que se reforman, adicionan y derogan diversas disposiciones del CFF, publicado el 9 de diciembre de 2013 en el Diario Oficial de la Federación, vigente en su mayoría, a partir del $1^{\circ}$ de enero de 2014, se desprende que la finalidad de las obligaciones antes mencionadas fue, supuestamente, simplificar y facilitar el cumplimiento de la obligación tributaria para los contribuyentes.

Asimismo, se mencionó que las reformas que nos ocupan tuvieron como objetivo: a) facilitar y simplificar los procedimientos de fiscalización; b) dotar de certeza jurídica al contribuyente; c) emplear los adelantos tecnológicos para una mejor comunicación entre el contribuyente y la autoridad fiscal, y d) brindar herramientas y mecanismos que procuren la eficiencia en el control y fiscalización.

En este sentido, es preciso señalar que si la finalidad de la obligación establecida en el artículo 28, fracción IV del CFF, es simplificar el cumplimiento de la obligación tributaria, así como emplear los adelantos tecnológicos para una mejor comunicación entre el contribuyente y la autoridad fiscal, las mismas de ninguna manera son adecuadas ni idóneas para alcanzar dicha finalidad.

Lo anterior, en virtud de que en la legislación actual existen medios a través de los cuales los contribuyentes cumplen con su obligación tributaria de manera simple, además de que están en constante comunicación con la autoridad fiscal, ya que se encuentran obligados a presentar declaraciones de impuestos mensuales y del ejercicio, así como informativas, de conformidad con las leyes fiscales correspondientes. Así, guisa de ejemplo, podemos citar los artículos 86 de la Ley del Impuesto sobre la Renta.

También, el artículo 32 de la Ley del Impuesto al Valor Agregado, establecen algunas de las obligaciones de las personas (morales), relativas a presentar declaraciones de impuestos de manera mensual, del ejercicio, así como informativas. En tal virtud, es claro que la obligación de ingresar de forma mensual la información contable a través de la página de Internet del SAT, establecida en el artículo 28, fracción IV del CFF, de ninguna manera es necesaria para "facilitar" el cumplimiento de las obligaciones 
tributarias, ni para mejorar la comunicación entre los contribuyentes y la autoridad fiscal, y menos, para impulsar tareas de gestión del tributo, ya que existen medios más adecuados e idóneos para ello, como la obligación de presentar declaraciones de impuestos e informativas establecidas en las leyes fiscales aplicables y de las cuales hemos dado cuenta con antelación.

Por otra parte, si la finalidad de las obligaciones establecidas en el artículo 28, fracción IV del CFF es brindar herramientas y mecanismos que procuren la eficiencia en el control y fiscalización, tampoco son adecuadas ni idóneas para alcanzar dicha finalidad, ya que de conformidad con lo establecido por el artículo 42 del CFF, la autoridad fiscal cuenta con una amplia gama de facultades de comprobación que puede practicar para alcanzar esta finalidad, además, la obligación prevista en el artículo 28, fracción IV del CFF no sólo no es adecuada e idónea para alcanzar el fin pretendido por el legislador, sino que implica una carga desmedida, excesiva e injustificada para los contribuyentes.

\section{CONCLUSIONES}

No obstante los tópicos que tratamos de abordar brevemente en páginas anteriores respecto de las implicaciones legales del envío de información contable por medios electrónicos a las autoridades hacendarias, queda claro, que los supuestos normativos de la legislación mexicana, mencionados en el presente trabajo, por un lado asisten con puntualidad a la cita con la globalización, pero de otro, dejan de lado la integración formal y material de los derechos y garantías del contribuyente al realizar lo que bien podríamos denominar el binomio "derecho-tecnología".

De igual manera, consideramos que hemos comprobado nuestra hipótesis, esto es, demostrar la manera en que se integraron al ordenamiento jurídico tributario las nuevas tecnologías, particularmente, la relativa a los medios electrónicos, las cuales, dicho sea de paso y como pudimos constatar, fueron aprobadas por el legislador mexicano sin mayor debate o análisis. Merece especial cita el hecho que hemos también verificado que la incorporación de medios electrónicos en el sistema jurídico para pretender regular la gestión del tributo en México trastoca de manera grave el artículo 16 de la CPEUM.

Un elemento adicional del cual también hemos podido dar cuenta, es que posiblemente, el artículo 28, fracción IV del CFF, viola el derecho humano a un sistema impositivo adecuado, justo y eficiente, toda vez que las autoridades responsables pretenden 
transferir al contribuyente una serie de obligaciones complejas, costosas y que le implican destinar un tiempo excesivo de su vida para que el SAT cuente con la contabilidad de los contribuyentes estructurada para futuros actos de fiscalización. Derechos humanos los antes mencionados, que se encuentran garantizados en lo artículos 26 de la Convención Americana Sobre Derechos Humanos (CADH) y 34 inciso c) y 45 incisos d) y e) de la Carta de la Organización de los Estados Americanos (COEA).

Debemos considerar en el presente apartado conclusivo, que el artículo 26 de la $\mathrm{CADH}$ dispone que los estados partes se comprometen a adoptar providencias, para lograr progresivamente la plena efectividad de los derechos que se derivan de las normas económicas y sociales contenidas en la COEA. De esta manera, tenemos que la igualdad de oportunidades, la eliminación de la pobreza crítica y la distribución equitativa de la riqueza y del ingreso, así como la plena participación de sus pueblos en las decisiones relativas a su propio desarrollo, son, entre otros, objetivos básicos del desarrollo integral. Para lograrlos, convienen asimismo en dedicar sus máximos esfuerzos a la consecución de metas básicas tales como: a) Sistemas impositivos adecuados y equitativos; b) Eficientes sistemas y procedimientos de consulta y colaboración entre los sectores de la producción, tomando en cuenta la protección de los intereses de toda la sociedad; y c) Funcionamiento de los sistemas de administración pública, banca y crédito, empresa, distribución y ventas, en forma que, en armonía con el sector privado, responda a los requerimientos e intereses de la comunidad;

En síntesis, las disposiciones de derecho internacional invocadas, conjuntamente con la CPEUM, contienen la obligación para el Estado Mexicano de establecer un sistema impositivo adecuado y eficiente que permita fomentar el crecimiento económico y social de sus habitantes, actuando de forma eficiente y, en consecuencia, evitando la transferencia de obligaciones onerosas propias del Estado al gobernado en perjuicio de sus garantías individuales.

En efecto, pese a las importantes y numerosas facultades de comprobación de que están legalmente dotadas las autoridades hacendarias $-y$ de las cuales hemos dado cuenta páginas atrás- no han bastado sin embargo para las autoridades fiscales, lo que demuestra en parte, un fenómeno de poca ineficacia administrativa, al cual, la solución simplista fue dotar todavía con mayores facultades para intervenir en la vida privada de las personas sin que medie acto de molestia por escrito en los términos que dicta nuestra CPEUM. 
Así, por último, podemos señalar también que las experiencias internacionales presentan, sin embargo, una clara tendencia a fortalecer mejor los derechos y garantías del contribuyente, como un medio más idóneo de incrementar la gestión tributaria, pues el marco de certeza jurídica y la consagración legal y sistemática de nuevos esquemas de defensa y protección de los contribuyentes han incidido de manera importante en un crecimiento real y efectivo de los ingresos tributarios, según esas mismas experiencias internacionales. 


\section{REFERENCIAS}

Carbonell Sánchez, M. (2013). Teoría de los derechos humanos, (2 ${ }^{\text {da }}$ Edición) (pp. 65 67). México: Universidad Nacional Autónoma de México-Instituto de Investigaciones Jurídica.

Carta de la Organización de los Estados Americanos.

Código Fiscal de La Federación. (2014). Última reforma publicada Diario Oficial de la Federación el 14 de marzo de 2014

Convención Americana Sobre Derechos Humanos.

Constitución Política de los Estados Unidos Mexicanos. (2014). Última reforma publicada en el Diario Oficial de la Federación el 07 de julio de 2014. México

Checa González, C. (2010). Globalización económica y principios tributarios. Revisa Mexicana de Derecho Constitucionales Cuestiones Constitucionales, (22), 79113.

Dugain, V. y Olaberria, E. (2015). What Makes Mexicans Happy?, OECD Economics Department Working Papers,(1196). Recuperado de http://dx.doi.org/10.1787/5js4h5qp6I0w-en

Fernández González, A. (julio-diciembre, 2012). Entes instrumentales en políticas de innovación tecnológica de las administraciones públicas: La experiencia de los centros de supercomputación en España y su comparación con otros países. Gestión y Análisis de políticas Públicas. Revista del Instituto Nacional de Administración Pública del Ministerio de Hacienda y Administraciones Pública de España, Nueva Época, (8), 65-97. Recuperado de http://revistasonline.inap.es/index.php?journal=GAPP\&page=article \&op=view\&p ath\%5B\%5D=10004\&path\%5B\%5D=10339

Fernández Pavés, MA. J. (1991). Administración y gestión tributaria. Comentarios a la Ley General Tributaria y líneas para su reforma. Homenaje a Fernando Sainz de Bujanda (pp. 1291-1308). Madrid: Instituto de Estudios Fiscales.

Ley del Impuesto al Valor Agregado. (2013). Última reforma publicada en el Diario Oficial de la Federación el 11 de diciembre de 2013.

Ley del Impuesto Sobre la Renta. (2013). Última reforma publicada en el Diario Oficial de la Federación el 11 de diciembre de 2013.

Ley Orgánica del Servicio de Administración Tributaria. (2012). Última reforma publicada en el Diario Oficial de la Federación el 09 de abril de 2012.

Ochoa Huera, C. (Enero-Abril, 2015). El carácter administrativo del derecho a la información. Boletín Mexicano de Derecho Comparado.

Organización para la cooperación y el desarrollo económico. (2014). Perspectivas de 
la OCDE sobre ciencia, tecnología e industria 2014 (Version abreviada): Informe Iberoamericano. México: Autor.

Pleno de la Suprema Corte de Justicia de la Nación. (1998). Fundamentación y Motivacion de los Actos de Autoridad Legislativa (Tesis Jurisprudencial). UNAM, México. Recuperado de http://info4.juridicas.unam.mx/const/tes/7/12/5825.htm

Pleno de la Suprema Corte de la Nación. (2007). Garantías Individuales. El Desarrollo de sus límites y la Regulación de sus posibles conflictos por parte del Legislador debe respetar los principios de Razonabilidad y Proporcionalidad Jurídica (Tesis Jurisprudencial). Tribunal Pleno, México D.F. Recuperado de https://www.scjn.gob.mx/transparencia/Documents/Inf\%200torgada\%20Jur/200 7/174_01.pdf

Ponce Solé, J. (Enero-Junio 2014). Ciencias sociales, Derecho Administrativo y buena gestión pública. De la lucha contra las inmunidades del poder a la batalla por un buen gobierno y una buena administración mediante un diálogo fructífero. Gestión y Análisis de políticas Públicas. Revista del Instituto Nacional de Administración Pública del Ministerio de Hacienda y Administraciones Pública de España, Nueva Época, 11. Recuperado de http://revistasonline.inap.es/index.php?journal=GAPP\&page=issue\&op=view\&p ath\%5B $\% 5 \mathrm{D}=685$

Téllez Valdéz, J.A. (2013). Lex cloud computing. Estudio jurídico del cómputo en la nube de México (p.17). México: Instituto de Investigaciones JurídicasUniversidad Nacional Autónoma de México. Recuperado de http://biblio.juridicas.unam.mx/libros/7/3249/4.pdf

Tribunales Colegiados de Circuito. (2009). Suspensión Definitiva en el Amparo. Cuestiones Jurídicas (Tesis Jurisprudencial). Semanario Judicial de la Federación y su Gaceta. Recuperado de http://sjf.scjn.gob.mx/sjfsist/Documentos/Tesis/163/163052.pdf

Tribunales Colegiados de Circuito. (2011). Posesión simple de tarjetas de crédito, débito o cualquier otro instrumento de pago. (Tesis Jurisprudencial). Semanario Judicial de la Federación y su Gaceta, México. Recuperado de http://sjf.scjn.gob.mx/sjfsist/Documentos/Tesis/163/163052.pdf

Tribunales Colegiados de Circuito. (2012). Apariencia del Buen Derecho. Cuestiones Jurídicas (Tesis Jurisprudencial). Semanario Judicial de la Federación y su Gaceta. Recuperado de http://200.38.163.178/sjfsist/Documentos/Tesis/2001/2001572.pdf Suprema Corte de Justicia de la Nación. (2015). Suspensión en el Juicio de Amparo 
(Tesis Jurisprudencial). Seminario Judicial de la Federación, Ciudad de México. Recuperado de http://sjf.scjn.gob.mx/SJFSem/Paginas/DetalleGeneralV2.aspx?Epoca=\&Apendi ce=\&Expresion=\&Dominio=Tesis\%20\%20publicadas\%20el\%20viernes\%2013 \%20de\%20febrero\%20de\%202015.\%20Segunda\%20Sala\&TA_TJ=1\&Orden=3 $\&$ Clase $=$ DetalleSemanarioBL $\&$ Tablero $=\&$ NumTE $=9 \& E p p=20 \&$ Desde $=-$ 100\&Hasta $=-$ 100\&Index=0\&Semanald=201507\&ID=2008430\&Hit=1\&IDs=2008430,2008429, 2008428,2008427,2008426,2008425,2008424,2008423,2008422\&Epoca=100\&Anio=-100\&Mes $=-100 \&$ Semanald $=201507 \&$ Instancia $=2 \& T A T J=1$ 